\title{
Lactic acid can be easily and precisely determined by reversed-phase high performance liquid chromatography with pre-column derivatization*
}

\author{
M. Czauderna ${ }^{1}$ and J. Kowalczyk \\ The Kielanowski Institute of Animal Physiology and Nutrition, Polish Academy of Sciences \\ 05-110 Jabtonna, Poland
}

(Received 2 February 2008; accepted 8 April 2008)

\begin{abstract}
Simple, sensitive and rapid methods of lactic acid (LA) quantification in the presence of other short-chain fatty acids in specimens of biological origin were developed to facilitate investigation of metabolism in body tissues and fluids of farm animals, fermentation processes, and ensiled products by reversed-phase high-performance liquid chromatography (RP-HPLC). LA and other acids in assayed samples were converted to their sodium salts (RCOONa). Derivatization of RCOONa was carried out with of a solution of 2,4'-dibromoacetophenone (DBAP) (2.2-2.4 g DBAP/100 ml of acetone or $20 \mathrm{~g} \mathrm{DBAP} / 100 \mathrm{ml}$ of chloroform) and triethylamine. The reaction mixture was vigorously mixed and then reacted for $1 \mathrm{~h}$ at $45^{\circ} \mathrm{C}$. The separation of derivatized acids was performed on a Nova Pak column $(4 \mu \mathrm{m}, 300 \times 3.9 \mathrm{~mm}$ I.D., Waters) using a binary gradient elution program and photodiode detection at $259 \mathrm{~nm}$. Lactic, acetic and propionic acid peaks were eluted at $11.05 \pm 0.10$, $18.0 \pm 0.1$ and $19.6 \pm 0.1 \mathrm{~min}$, respectively, while the iso-capronic acid peak (an internal standard) at $23.1 \pm 0.1 \mathrm{~min}$. The total run time of the HPLC analysis was $59 \mathrm{~min}$. LA detection at $259 \mathrm{~nm}$ assures the excellent selectivity and high sensitivity of the proposed method; the limit of detection (LOD) and the limit of quantification (LOQ) were equal to 0.04 and $0.13 \mathrm{ng} / \mathrm{ml}$, respectively. The use of chloroform as a solvent for DBAP in the derivatization of LA in samples followed by RP-HPLC offers the best sensitivity of LA determination in biological samples. The utility of the method was demonstrated by LA analysis in different materials such as silage, fermented dairy and vegetable products, intestinal digesta, blood and etc. The presented methods based on an inexpensive HPLC column, simple and rapid processing of samples provide accurate and sensitive analytical tools for routine determination of LA, particularly in specimens of biological origin.
\end{abstract}

KEY WORDS: lactic acid, determination, derivatization, biological samples, HPLC

\footnotetext{
${ }^{*}$ Supported in part by the Ministry of Science and Higher Education, Grant No. N N311 336433

${ }^{1}$ Corresponding author: e-mail: m.czauderna@ifzz.pan.pl
} 


\section{INTRODUCTION}

Lactic acid (LA) has many important biochemical and physiological properties. It is chiral and has two optical isomers: D-(-)-lactic acid ( $R$-lactic acid) and its mirror image, $\mathrm{L}-(+)$-lactic acid (S-lactic acid), which is the biologically important isomer (Ewaschuk et al., 2004). In animal bodies, L-LA is constantly synthesized from pyruvate via lactate dehydrogenase in a fermentation process during metabolism and, especially, during intensive exercise like sprinting. DL-LA is also an intermediate in animals, particularly ruminants (Ewaschuk et al., 2004; Davies et al., 2007). Formation of DL-LA from feed components is highly correlated with the sum of the soluble sugar and soluble protein content in forage (Weinberg et al., 2004a). Moreover, silage, a major component in the rations of dairy cattle, is forage preserved through lactic acid fermentation (Ricke, 2003). Therefore, inoculants comprising mainly lactic acid bacteria (LAB) are applied as silage supplements to stimulate ensiling fermentation. Some LAB strains produce via lactic acid an aromatic organic acid, phenyllactic acid, possessing fungicidal activity (Greifova et al., 2007).

Lactic acid, a product of fermentation of lactose, can also be found in many milk products (koumiss, kefir, yogurt or several cottage cheeses) as well as in ensiled vegetables, grasses, etc. LA can also be used as a food preservative or as a $\mathrm{pH}$ adjusting additive to various processed foods (Ricke, 2003).

Considering the above, monitoring concentrations of LA and other organic acids in specimens of biological origin is essential in physiological and nutritional studies. Therefore, titration after steam distillation, enzymatic, spectrometric, colorimetric and electrophoresis methods, chemiluminescence biosensor assay, gas (GC) and liquid (HPLC) chromatography have been used for the determination of LA and other shortchain fatty acids $\left(\mathrm{C}_{2}-\mathrm{C}_{6}\right)$ in specimens of biological origin (Gomez-Alvarez et al., 1999; Czauderna and Kowalczyk, 2001, 2002; Czauderna et al., 2001, 2002; Mataix et al., 2001; Ewaschuk et al., 2004; Wu et al., 2005; Resende et al., 2006; Inoue et al., 2007). The main advantages of high-resolution HPLC over the above-mentioned methods are simplicity, the possibility of using selective HPLC columns and very sensitive photodiode detection having excellent response linearity over a wide concentration range of acids in samples (Sivakesava et al., 2001; Lin and McKeon, 2005). Unfortunately, LA as well as other organic acids and their methyl/ethyl esters have high molar absorptions only at short UV wavelengths $(<205 \mathrm{~nm})$. Therefore, for quantification of LA in the presence of other volatile (VFA) and short-chain fatty acids (SCFA) by HPLC it is better to convert them with reagents having high molar absorptivity at longer UV wavelengths ( $>250 \mathrm{~nm}$ ) (Czauderna et al., 2002).

For these reasons it seemed desirable to develop simple and more selective derivatization methods for quantification of LA, particularly in the presence of other VFA and SCFA, using selective reversed-phase liquid chromatography (RP-HPLC) with sensitive photodiode array detection. To obtain satisfactory stability of the 
formed LA derivative we used 2,4'-dibromoacetophenone as the derivative reagent, and a long HPLC $\mathrm{C}_{18}$ column for the highly efficient resolution of LA from other acids and endogenous species present in biological samples.

\section{MATERIAL AND METHODS}

\section{Chemicals and materials}

All chemicals were of analytical grade and organic solvents were of HPLC grade. Lactic acid (LA), acetic acid (AA), propionic acid (PA) and chloroform were purchased from POCh (Gliwice, Poland), while iso-capronic acid (an internal standard; IS) was from Sigma (St. Louis, MO, USA). Methanol and acetonitrile were purchased from Lab-Scan (Eire), whereas 2,4'-dibromoacetophenone (DBAP) and triethylamine were from Merck (Darmstadt, Germany). Water used for the preparation of mobile phases (Table 1) and chemical reagents was prepared using an Elix ${ }^{\mathrm{TM}}$ water purification system (Millipore, Canada).

Table 1 . The binary gradient elution program used for analysis of lactic acid, acetic acid, propionic acid and iso-capronic acid in standards and specimens of biological origin (the column temperature: $39^{\circ} \mathrm{C}$ )

\begin{tabular}{cccc}
\hline \multirow{2}{*}{$\begin{array}{c}\text { Time } \\
\min \end{array}$} & $\begin{array}{c}\text { Flow rate } \\
\text { m1/min }\end{array}$ & \multicolumn{2}{c}{ Composition, \% } \\
\cline { 3 - 4 } & 0.8 & 39.8 & solvent B \\
\hline 0 & 0.8 & 39.8 & 60.2 \\
12 & 0.8 & 64.8 & 60.2 \\
15 & 1.0 & 64.8 & 35.2 \\
18 & 1.2 & 80.0 & 35.2 \\
20 & 1.75 & 80.0 & 20.0 \\
25 & 1.5 & 98.0 & 20.0 \\
27 & 2.1 & 98.0 & 2.0 \\
29.5 & 2.75 & 98.0 & 2.0 \\
46.5 & 2.0 & 98.0 & 2.0 \\
46.9 & 0.8 & 39.8 & 2.0 \\
48.5 & 0.8 & 39.8 & 60.2 \\
59.0 & & & 60.2 \\
\hline
\end{tabular}

\section{Chromatographic equipments}

An alliance separation module (model 2690, Waters) was equipped with a Waters 996 photodiode array detector (DAD) operated in the UV range from 195 to $400 \mathrm{~nm}$. Development of the gradient systems, collection of chromatograms, peak integrations and peak purity analyses (in the UV range from 230 to $290 \mathrm{~nm}$ ) were performed using a Pentium III computer (Compaq) and Millennium 2001 
software (version 2.15). Acid derivatives were quantified at $259 \mathrm{~nm}$. UV spectra of analytes in an eluate with a spectral resolution of $1.2 \mathrm{~nm}$ were recorded every second and were electronically stored on a computer hard disk. Fractionations and quantifications were performed on a Nova Pak $\mathrm{C}_{18}$ column $(4 \mu \mathrm{m}, 300 \times 3.9$ $\mathrm{mm}$ I.D., Waters) in conjunction with a Waters guard column of $10 \times 6 \mathrm{~mm}$ I.D. containing reversed-phase $\mathrm{C}_{18}(30-40 \mu \mathrm{m})$ pellicular packing material.

Table 2. Effectiveness of lactic acid peak $\left(\mathrm{S}_{\mathrm{n}}{ }^{\text {new }}\right)$ determination in the lactic acid standard and specimens of biological origin; injection volumes - $2.5 \mu 1$

\begin{tabular}{lcc}
\hline Analysed sample & $\begin{array}{c}\text { Peak area } \\
\mathrm{S}_{\mathrm{n}}^{\text {new }}\end{array}$ & $\begin{array}{c}\text { Ratio of } \\
\text { peak area/background }\end{array}$ \\
\hline & \multicolumn{1}{c}{ The new derivatization method } \\
Lactic acid standard $^{1}$ & 35254738 & $65.6 \times 10^{6}$ \\
Grass silage $^{2}$ & 225538 & $2.8 \times 10^{6}$ \\
Ovine rumen fluid $^{3}$ & 1358435 & $11.8 \times 10^{6}$ \\
Ovine rumen fluid $^{4}$ & 2410920 & $19.7 \times 10^{6}$ \\
Ovine plasma blood $^{5}$ & 642533 & $27.7 \times 10^{6}$ \\
Broiler caecum digesta $^{6}$ & 3823848 & $11.6 \times 10^{6}$ \\
Broiler ileum digesta $^{6}$ & 461564 & $0.75 \times 10^{6}$ \\
Kefir $^{7}$ & 14903777 & $30.7 \times 10^{6}$ \\
Fermented $^{8}$ cucumber & 4433715 & $3.1 \times 10^{6}$ \\
\multicolumn{1}{c}{ cabbage } & 7716713 & $24.2 \times 10^{6}$ \\
red beet & 1041875 & $22.3 \times 10^{6}$ \\
\hline
\end{tabular}

$11.07 \mathrm{mg}$ of LA was used for the derivatization procedure according to the new derivatization method; intra-assay and inter-assay coefficient of variations based on 3 repeated analyses were equal to 1.2 and $2.5 \%$, respectively

$2110 \mathrm{~g}$ of carefully grinded grass silage was extracted with $75 \mathrm{ml}$ of ethyl alcohol; the resulting mixture was centrifuged at $2000 \mathrm{~g}$ for $10 \mathrm{~min}$. The supernatant was alkalized with $6 \mathrm{M} \mathrm{NaOH}$ to $\sim \mathrm{pH} 9$. Then the resulting mixture was centrifuged at $2000 \mathrm{~g}$ for $10 \mathrm{~min}$. Finally the supernatant ethyl alcohol was removed under a stream of argon at $\sim 30^{\circ} \mathrm{C}$. Obtained residue was used for the derivatization procedure according to the new derivatization method

${ }^{3}$ sheep were fed standard forage without extra carbohydrate; $11 \mathrm{ml}$ of a rumen fluid was alkalized with $6 \mathrm{M} \mathrm{NaOH}$ to $\sim \mathrm{pH} 9$. Then the resulting mixture was centrifuged at $2000 \mathrm{~g}$ for $10 \mathrm{~min}$. Finally the supernatant water was removed under a stream of argon at $\sim 30^{\circ} \mathrm{C}$. Obtained residue in a vial was used for the derivatization procedure according to the new derivatization method

${ }^{4} 11 \mathrm{ml}$ of a rumen fluid from sheep were fed standard forage enriched in a carbohydrate $(10 \mathrm{~g} / \mathrm{kg}$ forage); fluid was processed according to the protocol described in the superscript 3

${ }^{5} 2 \mathrm{ml}$ of plasma was used for the derivatization procedure according to the new derivatization method

${ }^{6} 5 \mathrm{ml}$ of digesta from caecum or ileum of a broiler were mixed with $4 \mathrm{ml}$ of water and then acidified with $0.5 \mathrm{ml}$ of formic acid. The resulting mixture was centrifuged for $10 \mathrm{~min}$ at $\sim 5000 \mathrm{~g}$ and next the obtained supernatant was centrifuged for $15 \mathrm{~min}$ at $\sim 6000 \mathrm{~g}$. Finally, $0.6 \mathrm{ml}$ of obtained supernatant was used for the derivatization procedure according to the new derivatization method

$75 \mathrm{~g}$ of kefir were used for the derivatization procedure according to the new derivatization method

${ }^{8} 6 \mathrm{ml}$ of fermented cucumber, cabbage or red beet juice were used for the derivatization procedure according to the new derivatization method 


\section{Preparation of samples and derivatization of acids}

Specimens of biological origin (Table 2) with $10 \mu 1$ of IS solution ( 1 mg of IS in $50 \mu \mathrm{l}$ of acetone) were alkalized with $6 \mathrm{M} \mathrm{NaOH}$ to $\sim \mathrm{pH} 9$ and then the resulting mixtures were centrifuged at $2000 \mathrm{~g}$ for $\sim 10 \mathrm{~min}$. Supernatants were separated from solid residues and the supernatant water was removed under a stream of argon at $\sim 30^{\circ} \mathrm{C}$. Obtained residues in reacti-vials were used for derivatization with 2,4'-dibromoacetophenone (DBAP) (2.2-2.4 g DBAP/100 ml of acetone) and triethylamine. The derivatizing procedure for assayed LA, AA, PA and IS was the same as for biological samples.

\section{Analytical solvents and gradient elution systems}

Two HPLC grade solvents were used in chromatographic analysis of derivatized acids. Solvent B was water with $5 \%$ methanol (v/v), while solvent A was methanol. For analysis of all derivatized acids in standards and biological samples, a binary gradient elution program was used (Table 1). Analyses of derivatized acid in standards and biological samples were performed at a column temperature of $39^{\circ} \mathrm{C}$, while analysed samples in the autosampler were maintained at $30^{\circ} \mathrm{C}$. Injection volumes were $1-50 \mu 1$. The maximum pressure of the HPLC system was $39 \mathrm{MPa}$. The limits of detection (LOD) were calculated as a signal-to noise ratio of 3, while the limit of quantification (LOQ) was defined as 10 times the noise under a peak (Gratzfeld-Husgen and Schuster, 1994; Meyer, 1999).

LA, AA and PA in biological samples were identified by the retention time of processed standards injected separately or/and by adding standard solutions to biological samples. It is recommended that all derivatized samples be protected from light and stored at about $-20^{\circ} \mathrm{C}$ when not in use.

\section{RESULTS AND DISCUSSION}

The main aim of the present studies was to develop pre-column derivatization methods for quantification of LA in the presence of other short-chain organic acids in biological samples using high-resolution RP-HPLC with sensitive photodiode detection. Initially, all acids in assayed samples were converted to their sodium salts (RCOONa). Therefore, no loss of LA and, especially, volatile fatty acids was found in derivatized samples (Lin and McKeon, 2005). To obtain a satisfactory yield of product formation, the derivatization of sodium salts of LA and other assayed acids was carried out with an excess of 2,4'-dibromoaceto-phenone (DBAP) and triethylamine. Based on our recent studies, derivatization should be performed at a lower temperature than the original reaction (i.e. 80 or $100^{\circ} \mathrm{C}$ ) and 
for a shorter time - less than $2 \mathrm{~h}$ (Czauderna et al., 2001, 2002). It thus seemed reasonable to convert LA and other assayed acids at $\sim 50^{\circ} \mathrm{C}$ and for only $\sim 60 \mathrm{~min}$. Our preliminary results are in agreement with our previous studies (Czauderna et al., 2001, 2002) in which the yield of the acid derivatization reaction positively correlated with medium alkalinity. Therefore, to achieve a satisfactory yield of the derivatization procedure, the conversion of assayed acids was performed using 2,4 '-dibromoacetophenone and an excess of triethylamine. It is also recommended to add iso-capronic acid as an internal standard (IS) to assayed samples.

\section{New derivatization method}

Based on systematic investigations concerning optimization of the derivatization reaction and our recent studies (Czauderna et al., 2001, 2002) following new method for the derivatization of LA and other assayed acids as presented below is recommended.

To a residue in a reacti-vial (see section "Preparation of samples and derivatization of acids), $800 \mu \mathrm{l}$ of a solution of DBAP (2.2-2.4 g DBAP/100 ml of acetone) and $200 \mu \mathrm{l}$ of triethylamine are added together with vigorous mixing. The resulting solution in a sealed tube is again mixed and reacted for $60 \mathrm{~min}$ at $45^{\circ} \mathrm{C}$. All derivatized samples are protected from the light. The derivatization procedure for standards is the same as for biological materials. The resulting standard solutions $(2-50 \mu \mathrm{l})$ or processed biological sample solutions $(2-6 \mu \mathrm{l})$ are injected onto a $\mathrm{C}_{18}$ column (Nova Pak). All processed biological samples are protected from light and stored at about $-20^{\circ} \mathrm{C}$ until analysed. For the HPLC assay of LA and other acids, frozen samples should be re-warmed to $\sim 30^{\circ} \mathrm{C}$ to avoid the absorption and/or precipitation of analysed LA, AA, PA and the added internal standard (IS). To determine the yield of the acid separation followed by derivatization of acids, it is recommended to add IS to assayed standards and specimens of biological origin.

\section{Ultra-efficient derivatization method}

To facilitate more sensitive and accurate analyses of very low concentrations of LA in specimens of biological origin, the objective of the current study was also to further improve the yield of the derivatization reaction. Modifications to the new derivatization method were required, specifically to select the solvent for ultra-efficient derivatization of LA in biological samples. Based on systematic investigations concerning optimization of the solubility of DBAP and derivatization reaction products, we recommend chloroform as the solvent for the ultra-efficient derivatization of LA and other assayed acids. Considering the above, the residue was used for the ultra-efficient derivatization method as below is recommended. 
To a residue in a reacti-vial (see section "Preparation of samples and derivatization of acids), $800 \mu \mathrm{l}$ of a solution of DBAP (20 g DBAP/100 ml of chloroform) and $200 \mu 1$ of triethylamine are added together with vigorous mixing. The resulting solution in a sealed tube is mixed again and reacted for $60 \mathrm{~min}$ at $45^{\circ} \mathrm{C}$. All derivatized samples are protected from the light. The derivatization procedure for standards is the same as for biological materials. The resulting biological sample solutions are injected (1-2.5 $\mu \mathrm{l}$ ) onto a $\mathrm{C}_{18}$ column (Nova Pak). All processed biological samples are protected from light and stored at about $-20^{\circ} \mathrm{C}$ until analysed. For the HPLC assay of LA and other acids, frozen samples should be re-warmed to $\sim 20^{\circ} \mathrm{C}$ to avoid the absorption and/or precipitation of analysed LA, AA, PA and IS, especially in assayed acid standards. To determine the yield of the acid separation followed by derivation of acids, it is recommended to add IS to assayed standards and specimens of biological origin.

\section{Evaluation of the new derivatization method}

A binary gradient system (Table 1) composed of $\mathrm{H}_{2} \mathrm{O}$ in methanol was found to provide a wide range of solvent strengths and excellent baseline stability (Figure 1). LA, AA, PA and IS can be satisfactorily separated using a long column packed with a strongly hydrophobic silica-based bonded phase. Satisfactory peak shapes, close to symmetrical, were observed with sample elution times even up to $24 \mathrm{~min}$. The derivatized LA as well as other acids were substantially retained on the reversed-phase column and were distinct from background interference or from endogenous substances present in assayed biological samples (Figure 1). In our procedure the LA peak was eluted at $11.05 \pm 0.10 \mathrm{~min}$, the AA peak at $18.0 \pm 0.1 \mathrm{~min}$, the PA peak at $19.6 \pm 0.1 \mathrm{~min}$, while the IS peak at $23.1 \pm 0.1$ min. As expected, the peaks of LA, AA, PA and IS (monitoring the yield of the procedure) were absent from the blank when the proposed gradient elution program and photodiode detections at 205 and $259 \mathrm{~nm}$ were used. Moreover, the responses of the photodiode array detector to the concentrations of LA, AA and PA in standards were linear functions (Figure 1). Indeed, as illustrated in Figure $1 \mathrm{~A}$, the relationship between the LA concentrations $\left(\mathrm{y}_{\mu \mathrm{g} / \mathrm{mm}}\right)$ and LA peak areas $\left(\mathrm{S}_{\mathrm{n}}\right)$ was linear over a wide range of concentrations of LA in standards (i.e. from 5 to $970 \mu \mathrm{g} \mathrm{LA} / \mathrm{ml})$, so, the correlation coefficient $\left(\mathrm{r}_{\mathrm{LA}}\right)$ was equal to 0.990 . Indeed, the use of monitoring at the maximum of LA derivative absorption assures excellent selectivity and high sensitivity of the proposed method. Consequently, the LOD and LOQ values obtained by the use of detection at $259 \mathrm{~nm}$ were equal to 0.04 and $0.13 \mathrm{ng} / \mathrm{ml}$, respectively. Chromatographic analyses (Figure 1, Table 2) evidenced that background fluctuations and the presence of endogenous species cannot interfere in the accurate and precise integration of the LA peak in all assayed specimens of biological origin. Analyses of LA purity and peaks based on 

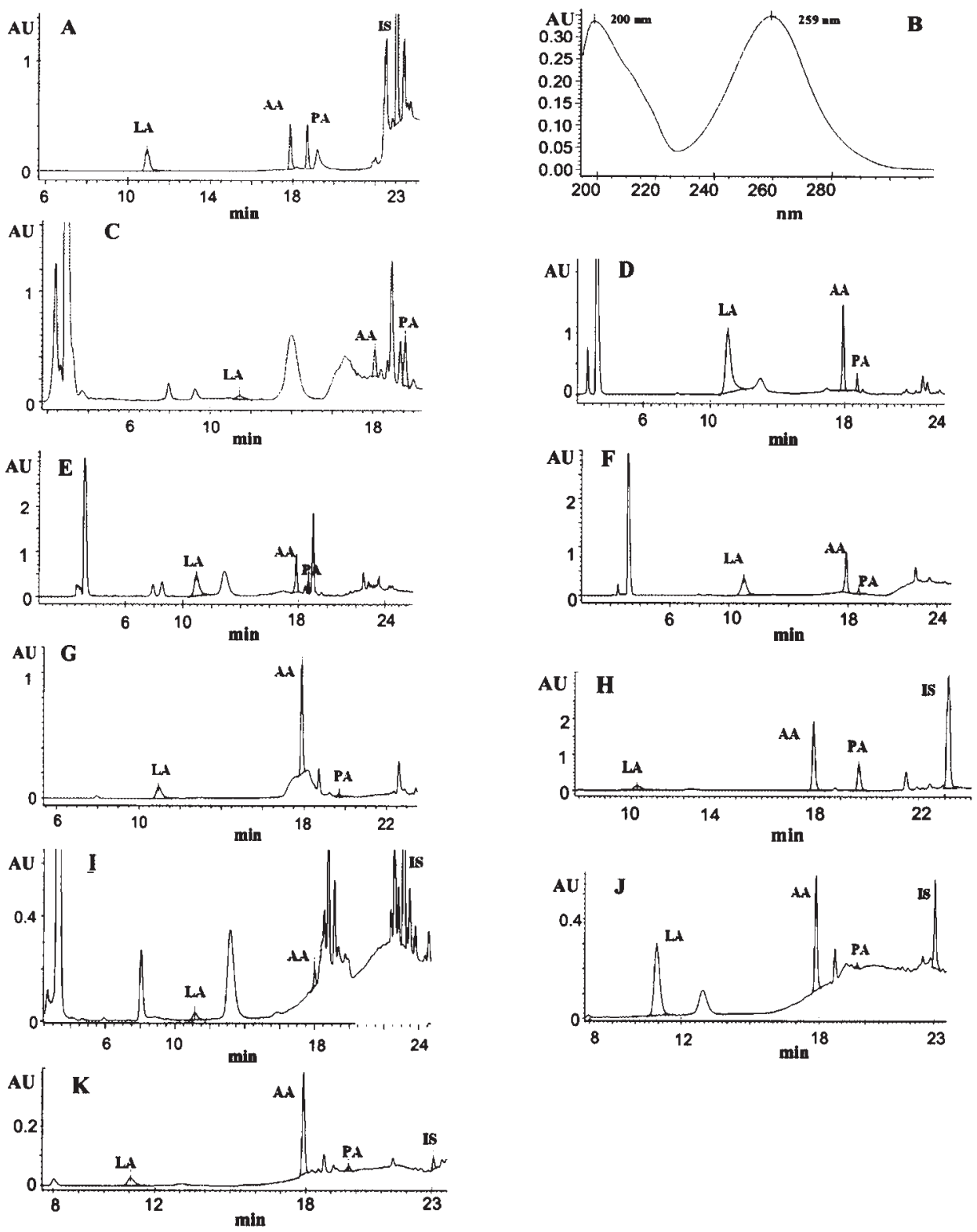

Figure 1. A - a chromatogram for derivatized lactic acid (LA), acetic acid (AA), propionic acid (PA) and iso-capronic acid (IS) using the new derivatization method and the elution program (Table 1; the detection at $259 \mathrm{~nm}$; AU - an absorption unit). B - UV spectra with their maxima $\left(\lambda_{\max }\right)$ of the derivatized standards of LA, AA, PA and IS. Chromatograms for derivatized acids in: $\mathrm{C}$ - grass silage; D - kefir; fermented: E - cucumber, F - cabbage G - red beet; H - ovine ruminal fluid; I - ovine blood plasma; J - broiler caecum digesta; $\mathrm{K}$ - broiler ileum digesta. Injection volumes: 2-6 $\mu \mathrm{l}$ 
relationships between the monitoring wavelength $(\lambda)$ and the LA peak area $\left(S_{n}\right)$ in assayed biological samples ( $\mathrm{S}_{\mathrm{n}}^{\text {sample }}$ ) and the LA standard $\left(\mathrm{S}_{\mathrm{n}}{ }^{\text {standard }}\right.$ ) (Czauderna and Kowalczyk, 2001, 2002) indicate that in all assayed biological samples, the LA and IS peaks are pure $(\sim 100 \%)$ in the UV range from 230 to $290 \mathrm{~nm}$. Detection at $259 \mathrm{~nm}$ provides the greatest response of the detector (i.e. $\mathrm{S}_{\mathrm{n}} /$ background) as compared with detection at a shorter wavelength $(\lambda \leq 244 \mathrm{~nm})$. Moreover, LA and, particularly AA, PA and IS monitoring at a short UV wavelength $(\lambda \leq 225 \mathrm{~nm})$ resulted in a significant decrease in the signal-to-noise ratio and/or an increase in interference from endogenous species present, particularly in rumen fluid, caecum and ileum digesta, silage, kefir and fluids from ensiled cucumbers or cabbage.

\section{Comparison of the new derivatization method with the ultra-efficient derivatization method}

The key step in the ultra-efficient derivatization method is complete dissolution of a high amount of derivatizing reagent (20 g DBAP in $100 \mathrm{ml}$ of chloroform) and forming derivatives of LA, AA, PA and IS as well as all endogenous substances present in analysed biological samples. Fortunately, chloroform is an excellent solvent for the derivatizing reagent, acid derivatives, and endogenous substances of assayed biological samples containing trace or high concentrations of LA. As can be seen from detailed chromatographic studies of the ultra-efficient derivatization method, particularly excellent fractionation of LA and IS from endogenous species of samples was achieved using the proposed binary gradient elution program (Table 1). Based on the UV spectra of assayed acid derivatives formed in the chloroform medium, we can conclude that the ultra-efficient derivatization method was also found to produce acid derivatives possessing high molar absorption with a maximum at $259 \mathrm{~nm}$. Indeed, excellent, nearly symmetrical LA, AA, PA and IS peak shapes, and satisfactory separation of these derivatives from other endogenous substances were observed with sample elution times even up to 24 min (Figure 2). Moreover, LA, and IS derivative peak areas in chromatograms of samples processed using the ultra-efficient derivatization method are considerably larger than the peak areas of these acids in samples processed using the current new derivatization method (Figure 2, Table 3). Considering the above, it can be concluded that a greater yield of acid derivatives can be achieved using the ultraefficient derivatization method compared with the new derivatization method.

The reproducibility of acid derivative analyses was examined in detail with respect to the storage of processed samples at $-20^{\circ} \mathrm{C}$ for $24 \mathrm{~h}$. The obtained results of chromatographic analyses documented that areas of LA and IS monitored at $259 \mathrm{~nm}$ were practically constant when samples were processed by the ultra-efficient derivatization method and warmed to only $\sim 20^{\circ} \mathrm{C}$ before HPLC analysis. Purity analyses of LA and LA peaks based on relationships between the monitoring 

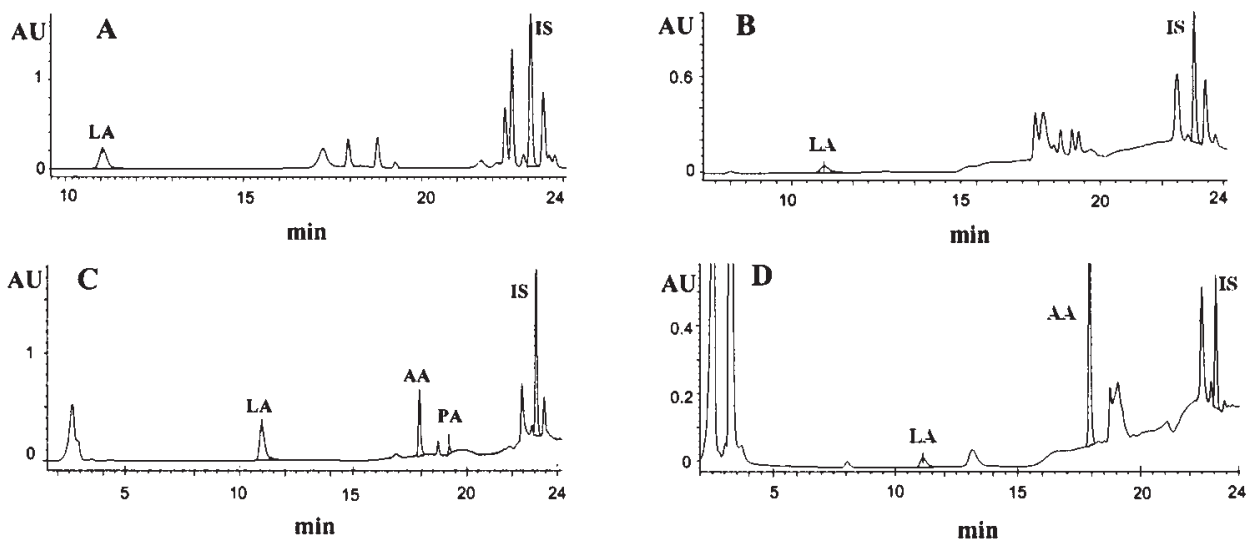

Figure 2. Dependence of the yield derivatization of the same amounts of LA and IS standards upon the derivatization procedure: A - the ultra-efficient derivatization method; B - the new derivatization method. Parts of a typical chromatogram for derivatized acids in $1 \mathrm{~g}$ of kefir processed using the ultra-efficient derivatization method (the chromatogram C) and the new derivatization method (the chromatogram D). All derivatized acids were separated using the binary gradient elution program (Table 1) and the UV detection at $259 \mathrm{~nm}$. Injection volumes $2.5 \mu 1$

Table 3. Comparison of the effectiveness of lactic acid (LA) determination in the LA standard ${ }^{1}$ and in kefir using the new derivatization method $\left(\mathrm{S}_{\mathrm{n}}{ }^{\text {new }}\right)^{2}$ and the ultra-efficient derivatization method $\left(\mathrm{S}_{\mathrm{n}} \text { ultra }\right)^{3}$

\begin{tabular}{lccc}
\hline Analysed & \multicolumn{3}{c}{ Ratio of } \\
\cline { 2 - 4 } sample & $\mathrm{S}_{\mathrm{n}}^{\text {ultra } / \mathrm{S}_{\mathrm{n}} \text { new }}$ & $\mathrm{S}_{\mathrm{n}}$ ultra $/$ background & $\mathrm{S}_{\mathrm{n}}{ }^{\text {new }} /$ background \\
\hline LA standard & $3.73 \pm 0.11$ & $327 \times 10^{6}$ & $49.7 \times 10^{6}$ \\
Kefir & $3.55 \pm 0.13$ & $191 \times 10^{6}$ & $18.5 \times 10^{6}$ \\
\hline
\end{tabular}

${ }^{1} 0.25 \mathrm{mg}$ of LA was used for the derivatization procedure according to the new derivatization method and the ultra-efficient derivatization method

${ }^{2}$ the inter-day coefficient variations based on 3 repeated analyses - 3.1\% ( $1 \mathrm{~g}$ kefir samples were stored for $24 \mathrm{~h}$ at $-20^{\circ} \mathrm{C}$ )

${ }^{3}$ the inter-day coefficient variations based on 4 repeated analyses $-2.7 \%$ ( $1 \mathrm{~g}$ kefir samples were stored for $24 \mathrm{~h}$ at $-20^{\circ} \mathrm{C}$ )

${ }^{4}$ the limit of detection (LOD) $-0.014 \mathrm{ng} / \mathrm{ml}$; the limit of quantification (LOQ) $-0.046 \mathrm{ng} / \mathrm{ml}$

wavelength and the LA peak area $\left(\mathrm{S}_{\mathrm{n}}\right)$ in assayed biological samples $\left(\mathrm{S}_{\mathrm{n}}\right.$ sample $)$ and the LA standard ( $\mathrm{S}_{\mathrm{n}}$ standard) also indicate that in the assayed biological samples the LA and IS peaks are pure ( 100\%) in the UV range from 242 to $278 \mathrm{~nm}$. Thus, the above results documented that the insolubility and/or adsorption of acid derivatives in chloroform medium is lower than in acetone medium (i.e. the solvent used for processing samples according to the new method of derivatization). Therefore, the ultra-efficient derivatization method provides a very accurate and sensitive (LOD: $0.014 \mathrm{ng} / \mathrm{ml}$; LOQ: $0.046 \mathrm{ng} / \mathrm{ml}$ ) analytical tool for routine determination 
of very low concentrations of LA in specimens of biological origin. This method is also recommended for small samples $(<1 \mathrm{~g})$ analysed in order to quantify LA.

\section{CONCLUSIONS}

The proposed new and ultra-efficient derivatization methods are universal and simple analytical tools for accurate and precise determination of lactic acid (LA) in particular, as well as acetic acid (AA), propionic acid (PA), in various types of biological samples. LA, AA, PA and iso-capronic acid (IS) derivatives were satisfactorily separated using widely available and inexpensive $\mathrm{C}_{18}$ columns and a typical UV detector. The total run time of HPLC analyses including the conditioning and equilibration was $59 \mathrm{~min}$. The use of derivatives possessing a chromophoric group responsible for the very high molar absorptivity make these compounds almost ideally suited for RP-HPLC with detection at a relatively transparent UV region (i.e. at $259 \mathrm{~nm}$ ). Moreover, due to aromatic derivatization, these acids are substantially retained on the $\mathrm{C}_{18}$ column compared with underivatized acids analysed by liquid- or gas-chromatography. The use of our liquid chromatography is more rapid, simple and selective than colorimetric or enzymatic methods, chemiluminescence biosensor assay, or titration after steam distillation.

The use of the ultra-efficient derivatization method followed by high-resolution RP-HPLC in conjunction with selective UV detection at $259 \mathrm{~nm}$ offers the best accuracy and sensitivity for the determination of LA in biological samples. Considering large LA peak areas in all assayed biological samples and small injection volumes (1-6 $\mu 1)$, we claimed that small amounts of biological materials $(\sim 100 \mathrm{mg})$ are sufficient for the LA analysis using the new derivatization method or particularly the ultra-efficient derivatization method followed by RP-HPLC with DAD.

\section{REFERENCES}

Czauderna M., Kowalczyk J., 2001. Separation of some mono-, di- and tri-unsaturated fatty acids containing 18 carbon atoms by HPLC and photodiode detection. J. Chromatogr. B 760, 165-178

Czauderna M., Kowalczyk J., 2002. HPLC separation of some unsaturated and saturated fatty acids. Chem. Anal. (Warsaw) 47, 867-882

Czauderna M., Kowalczyk J., Chojecki G., 2001. An improved method for derivatization of fatty acids for liquid chromatography. J. Anim. Feed Sci. 10, Suppl. 2, 369-375

Czauderna M., Kowalczyk J., Niedźwiedzka K.M., Wąsowska I., 2002. High efficient method for derivatization of fatty acids for high performance liquid chromatography. J. Anim. Feed Sci. 11, 517-526

Davies D., Theodorou M., Newbold J., 2007. Biotransformation and fermentation - exploiting the rumen and silo. IGER Inovations (Aberytwyth), No. 11, 37-41 
Ewaschuk J.B., Naylor J.M., Barabash W.A., Zello G.A., 2004. High-performance liquid chromatographic assay of lactic, pyruvic and acetic acids and lactic acid stereoisomers in calf feces, rumen fluid and urine. J. Chromatogr. B, 805, 347-351

Gomez-Alvarez E., Luque-Perez E., Ros A., Valcarcel M., 1999. Flow injection spectrophotometric determination of lactic acid in skimmed milk based on a photochemical reaction. Talanta 50, 121-131

Gratzfeld-Husgen A., Schuster R., 1994. HPLC for Environmental Analysis, Hewlett-Packard (France)

Greifova M., Krajcova E., Greif G., Gajdosova Z., Karovicova J., Schmidt S., 2007. Protective character of lactic acid bacteria. Folia Vet. 2, 84-85

Inoue Y., Shinka T., Ohse M., Kohno M., Konuma K., Ikawa H., Kuhara T., 2007. Changes in urinary level and configuration ratio of D-lactic acid in patients with short bowel syndrome. J. Chromatogr. B 855, 109-114

Lin J.-T., McKeon T.A., 2005. HPLC of Acyl Lipids. HNB Publishing, New York

Mataix E., Luque de Castro M.D., 2001. Determination of 1-(-)-malic acid and 1-(+)-lactic acid in wine by a flow injection-dialysis-enzymic derivatisation. Anal. Chim. Acta, 428, 7-14

Meyer V.R., 1999. Practical High-Performance Liquid Chromatography. Wiley, Chichester (UK)

Resende Jr. J.C., Pereira M.N., Bôer H., Tamminga S., 2006. Comparison of techniques to determination the clearance of ruminal volatile fatty acids. J. Dairy Sci. 89, 3096-3106

Ricke S.C., 2003. Perspectives on the use of organic acids and short chain fatty acids as anti-microbials. Poultry Sci. 82, 632-639

Sivakesava S., Irudayaraj J., Ali D., 2001. Simultaneous determination of multiple components in lactic acid fermentation using FT-MIR, NIR, and FT-Raman. Process Biochem. 37, 371-378

Weinberg Z.G., Chen Y., Gamburg M., 2004. The passage of lactic acid bacteria from silage into rumen fluid, in vitro studies. J. Dairy Sci. 87, 3386-3397

Wu F., Huang Y., Huang C., 2005. Chemiluminescence biosensor system for lactic acid using natural animal tissue as recognition element. Biosens. Bioelectron. 21, 518-522 